\title{
Salud mental en menores españoles. Variables socioeducativas
}

\author{
Fernando Fajardo Bullón,' Benito León del Barco, ' Elena Felipe Castaño, 'María Isabel Polo del Río, ' \\ Eduardo Joao Ribeiro Dos Santos ${ }^{2}$
}

Artículo original

\section{ABSTRACT}

\section{Background}

Research on minors' mental health is a current necessity as a way to detect, analyze and prevent possible mental disorders.

\section{Objective}

The goal of this study was to analyze the influence of minors' educational stage and their parents' occupational social class as risk factors for minors' mental health.

\section{Method}

We used the results obtained in the National Spanish Health Survey 2006 , with a sample of 5812 minors between 4 and 15 years of age. Measures associated with the independent variables were estimated through the measurement of mental health on the Strengths and Difficulties Questionnaire (SDQ-parents' version).

\section{Results}

The relation between mental health and the variables occupational social class 1 (more privileged) (O.R. 0.256) and social class 2 (middle class) (O.R. 0.523) was significant $(p<0.05)$ in comparison with social class 3 (more disadvantaged). However, whether the minors were in pre-school (O.R. 1.138) or primary school (O.R. 1.162) was nonsignificant $(p>0.05)$ in comparison with secondary school.

\section{Discussion and conclusion}

It is concluded that high and middle occupational social classes are protector factors for minors' mental health in comparison to belonging to a low occupational social class. However, being a student in preschool or in primary school is not a risk factor for mental health in comparison to being a secondary school student.

Key words: Mental health, child welfare, social class, educational status.

\section{RESUMEN}

\section{Antecedentes}

La investigación de la salud mental en los menores de edad supone una necesidad actual como medida de detección, análisis y prevención de posibles trastornos mentales.

\section{Objetivo}

Analizar la influencia de la clase social ocupacional de los padres y de la etapa educativa en la que se encuentran los menores, como factores de riesgo en la salud mental de los menores españoles.

\section{Método}

Se utilizaron los resultados obtenidos en la Encuesta Nacional de Salud Española 2006, con una muestra de 5812 menores de cuatro a 15 años. Se estimaron las medidas de asociación con respecto a las variables independientes midiendo la salud mental mediante el Cuestionario de Capacidades y Dificultades (SDQ-versión padres).

\section{Resultados}

La relación entre la salud mental y la variable clase social ocupacional 1 (más privilegiadas) (OR 0.256) y la clase social 2 (clase media) (OR 0.523 ) aportaron valores significativos $(\mathrm{p}<0.05)$ con respecto a la clase social 3, más desfavorecida. Sin embargo, la pertenencia de los menores a la E. Infantil (OR 1.138) o a la E. Primaria (OR $1.162)$ no aportó valores significativos $(p>0.05)$ con respecto a la E. Secundaria.

\section{Discusión y conclusión}

Se puede concluir que las clases sociales ocupacionales elevadas y medias son factores protectores con respecto a la salud mental de los menores en comparación con la pertenencia a una clase social ocupacional baja. Sin embargo, la pertenencia a la E. Infantil o a la E. Primaria no supone un factor de riesgo de salud mental en comparación con la pertenencia a la E. Secundaria.

Palabras claves: Salud mental, bienestar del niño, clase social, nivel educativo.

Departamento de Psicología. Universidad de Extremadura. España.

2 Facultad de Psicología y Ciencias de la Educación. Universidad de Coímbra. Portugal

Correspondencia: Fernando Fajardo Bullón. Departamento de Psicología. Avenida de la Universidad s/n. Cáceres. 10071. Universidad de Extremadura. Extremadura, España. Tel: (92) 725 - 7664. E-mail: fernandofajardo@unex.es

Recibido primera versión: 18 de octubre de 2013. Segunda versión: 10 de noviembre de 2014. Aceptado: 1 de abril de 2015. 


\section{ANTECEDENTES}

El estatus socioeconómico y el nivel educativo han sido variables de amplio interés en investigación para analizar la salud de las personas a lo largo de las diferentes etapas evolutivas. ${ }^{1,2}$ Diversos estudios internacionales han puesto de relieve la relación entre un nivel alto de ingresos económicos y una buena salud autopercibida en adultos, tanto en hombres como en mujeres de diferentes países., ${ }^{3,4}$ Este tema ha cobrado gran relevancia al demostrarse que las diferencias económicas pueden llevar a diferencias de estatus social y a la exclusión social, lo que en sí mismo puede generar diferencias en la salud global., ${ }^{5,6}$ De hecho, en Norte América los trastornos de ansiedad y depresión aparecen doblemente en los grupos de bajos ingresos y bajos niveles educativos en comparación con aquellos de mayores ingresos y nivel educativo. ${ }^{7,8}$

Si nos centramos en los menores, las familias con un bajo nivel socioeconómico se asocian con niveles bajos de salud en adolescentes hasta los 18 años, siendo significativa esta asociación en todas las edades aunque con una mayor consistencia en la etapa de la adolescencia. ${ }^{9}$ De esta manera, aquellos menores con padres con bajos ingresos y bajo estatus socioeconómico, pueden llegar a duplicar la probabilidad de padecer problemas de salud y llegar a influir en la mayor probabilidad de aparición de comportamientos de riesgo en la adolescencia. ${ }^{10} \mathrm{Si}$ se atiende específicamente a la salud mental de los menores, se ha demostrado que entre el $3 \%$ y el 18\% de las personas en la etapa infantil y adolescente sufren algún tipo de trastorno mental, ${ }^{11-13}$ comprendiendo esta cifra entre el 10\% y el $20 \%$ en España. ${ }^{14}$ Ante estos datos es importante conocer si el nivel educativo del menor y la clase social ocupacional de los padres pueden estar relacionados con la presencia de problemas de salud mental en los menores españoles.

Para la medición de dichas patologías se ha utilizado el Strengths and Difficulties Questionnaire (SDQ) $\cdot{ }^{15}$ El SDQ es el instrumento más ampliamente utilizado en investigación en salud mental infantil desde 1997. ${ }^{16,17}$ Ha sido utilizado para encuestas nacionales de salud de diversos países ${ }^{18}$ demostrando sus propiedades psicométricas en diferentes culturas. ${ }^{19}$ Se puede utilizar en tres modalidades diferentes, según la persona que responda al cuestionario: profesores, padres o autoinforme. Es una herramienta de cribado muy utilizada frente a otras clásicas de diagnóstico más extensas como el CBCL o la escala de Conners. ${ }^{16,20,21}$ La versión española fue validada por Rodriguez ${ }^{22}$ y analizada con población nacional en otras investigaciones. ${ }^{23}$ Por tanto, el objetivo de este estudio fue analizar, mediante el SDQ-padres, la presencia de problemas mentales en menores españoles de cuatro a 15 años según la influencia del nivel educativo correspondiente a la edad y la clase social ocupacional de los padres.

\section{MÉTODO}

\section{Diseño del estudio}

Los datos empleados procedieron de la Encuesta Nacional de Salud Española 2006/2007 (ENSE-2006). Se realizó un estudio transversal en el que se seleccionaron 5812 menores, con un $50.8 \%$ de chicos y un $49.2 \%$ de chicas, con edades entre cuatro y 15 años. La información se obtuvo mediante el SDQ-versión padres y otras preguntas sociodemográficas (clase social ocupacional del principal sustentador familiar y ciclo educativo correspondiente a la edad del menor) integradas dentro del Cuestionario de Menores de la ENSE2006. La población de estudio de la ENSE-2006 fue seleccionada mediante un muestreo polietápico estratificado. Las respuestas se obtuvieron por medio de los principales sustentadores familiares de 31000 viviendas representativas de las 17 comunidades autónomas y extendidas por todo el territorio español. Una información más amplia se puede encontrar en la Encuesta Nacional de Salud-2006. ${ }^{24}$

Desgraciadamente, el cuestionario SDQ solamente se ha utilizado en la encuesta de salud española del año 2006. Por todo ello, el análisis de la base de datos de la ENSE -2006 supone una oportunidad única y exclusiva para poder analizar la salud mental de los menores españoles de cuatro a 15 años por medio de esta prueba utilizada internacionalmente.

\section{Variables}

El instrumento de medida SDQ-versión padres, diseñado por Goodman en el Instituto de Psiquiatría de Londres, ${ }^{15}$ ha sido traducido a 66 idiomas (www.sdqinfo.com) y validado en varias ocasiones a nivel internacional. ${ }^{25-27}$ Es un instrumento breve, excelente para el cribado de trastornos de salud mental en menores ${ }^{17,25}$ con una consistencia interna destacada en todas sus escalas. ${ }^{28}$ Está formado por 25 ítems divididos en cinco escalas (síntomas emocionales, problemas conducta, hiperactividad, problemas compañeros y conducta prosocial), con una puntuación variable desde 0 hasta 10 puntos por escala. La puntuación total se obtuvo mediante la suma de las cuatro escalas sin incluir la escala prosocial. Esta supresión de la quinta escala se realizó para obtener la puntuación total de dificultades, tal y como se ha establecido en la metodología de diferentes investigaciones con el SDQ, entre las que se encuentra su creador. ${ }^{29,30}$ Una vez cuantificadas las respuestas, se obtuvieron las puntuaciones alcanzadas por cada menor en cada escala y la puntuación total variando entre 0 y 40 puntos. Para analizar la variable salud mental, ésta se dividió en dos categorías: padecer o no padecer problemas de salud mental según la puntuación total superior o igual a 20 puntos (padecer) o inferior a 20 puntos (no padecer), obtenida en la validación del SDQ-padres para población española. ${ }^{22,23}$ Esta información 
se complementó mediante una entrevista estructurada que recogió las siguientes variables: la clase social ocupacional del principal sustentador y el nivel educativo en el que se encuentra el menor según su edad correspondiente.

Se consideraron seis clases sociales ocupacionales, de acuerdo con la adaptación española de la clasificación del British Registrar General. ${ }^{31}$ Para el estudio de las variables de una manera más efectiva, los autores las agruparon en tres clases sociales: La clase 1 (agrupó las clases sociales más privilegiadas I y II), la clase 2 (agrupó a las clases medias III y IVa) y la clase 3 (agrupó a las clases menos privilegiadas de dicho registro $\mathrm{IVb}$ y $\mathrm{V}$ ).

Por otro lado, atendiendo a la edad de los menores, se han considerado tres niveles educativos correspondientes: Enseñanza infantil (menores entre cuatro y cinco años), Enseñanza primaria (menores entre seis y 12 años) y Enseñanza secundaria (menores entre 13 y 15 años).

\section{Análisis estadístico}

Para el análisis estadístico de la puntuación total obtenida en el test SDQ-padres y las variables o factores clase ocupacional y nivel educativo, se utilizaron técnicas de análisis de la varianza (ANOVA), mientras que para el análisis de la asociación entre padecer o no problemas de salud mental con respecto al nivel ocupacional y nivel educativo se utilizaron las herramientas estadísticas de razón de odds (odds ratio, $\mathrm{OR}$ ) y los riesgos relativos (relative risk o risk ratio, $\mathrm{RR}$ ). En este último análisis se utilizó la clase social ocupacional 3 (menos privilegiada) y la Enseñanza secundaria como clases comparativas de control. Para los cálculos estadísticos se utilizó el programa estadístico SPSS 19.0.

\section{RESULTADOS}

\section{Estadística descriptiva e inferencial}

La distribución frecuencial de la muestra en estudio, según las variables nivel ocupacional y nivel educativo de los menores, se puede observar en el cuadro 1 . El análisis de la varianza para un factor señaló la existencia de diferencias significativas entre las medias en la puntuación total en el SDQ-padres y las clases ocupacionales ( $F=44.260$; $\mathrm{p}<0.05)$. Mediante varios análisis post hoc (Bonferroni, Scheffè, Tamhane) se encontraron diferencias de medias entre todas las categorías, tomadas de dos en dos. Sin embargo, no se obtuvieron diferencias significativas entre las medias en la puntuación total en el SDQ-padres y los niveles educativos de los menores $(F=1.226 ; \mathrm{p}=0.293>0.05)$.

\section{Análisis de riesgos relativos ( $R R$ ) y odds ratio (OR)}

Una vez analizadas las diferencias de medias se analizaron los RR y OR tomando la Clase Social Ocupacional 3 y la Enseñanza secundaria como clases de control para las variables clase ocupacional y nivel educativo, respectivamente. Respecto a la variable dependiente, padecer o no problemas de salud mental, se utilizó la clasificación anteriormente indicada según la puntuación total superior (padecer) o inferior e igual a 20 puntos (no padecer).

1. Clase social ocupacional. En primer lugar se contrastó la existencia o no de independencia entre la clase social ocupacional y la salud mental. Se obtuvo una Chi-cuadrada de Pearson de 47.86 ( $\mathrm{p}<0.00)$, lo que indicó la existencia de una asociación entre ambas variables. Una vez asumida dicha asociación se midieron los RR y OR de los niveles de las variables estudiadas.

Entre la clase social 1 (más privilegiada) y la clase social 3 (menos privilegiada), se obtuvieron los siguientes datos, $\mathrm{RR}=0.476$ y OR=0.309. Los límites de los ratios, al 95\% de confianza, no contenían la unidad y las Chi-cuadradas de Pearson $=36.79$ y de Yates $=35.73$ confirmaron la significancia de estos resultados $(p=0.000<0.05)$. De este modo, el factor de riesgo clase social 1 fue un factor protector con respecto a la clase 3 para la presencia de problemas de salud mental (cuadro 2).

De igual forma, al comparar la clase 2 (clase media) con la clase 3 (clases menos privilegiadas) se obtuvieron $\mathrm{RR}=0.750$ y $\mathrm{OR}=0.476$. Los límites de los ratios, al 95\% de confianza, no contenían la unidad y las Chi-cua-

Cuadro 1. Puntuaciones medias en el SDQ-padres según la clase social ocupacional de los padres y el nivel educativo de los menores

\begin{tabular}{lrccc}
\hline Factores/variables & \multicolumn{3}{c}{$\begin{array}{c}\text { Puntuación media } \\
\text { SDQ-padres }\end{array}$} & Ds \\
\hline Clase ocupacional 1 & $\mathrm{N}$ & 5.78 & 5.71 \\
Clase ocupacional 2 & $1363(23.5 \%)$ & 6.94 & 6.23 \\
Clase ocupacional 3 & $3065(52.7 \%)$ & 8.04 & 6.96 \\
Puntuación total clase ocupacional & $5812(100.0 \%)$ & 6.93 & 6.34 \\
Educación infantil & $883(15.2 \%)$ & 6.79 & 6.47 \\
Educación primaria & $3208(55.2 \%)$ & 7.05 & 6.42 \\
Educación secundaria & $1721(29.6 \%)$ & 6.78 & 6.14 \\
Puntuación total educación & $5812(100.0 \%)$ & 6.93 & 6.34 \\
\hline
\end{tabular}


Fajardo Bullón et al.

Cuadro 2. Riesgos relativos y Odds Ratio en función de las diferentes variables estudiadas

\begin{tabular}{|c|c|c|c|c|c|c|c|}
\hline \multirow{2}{*}{$\frac{\text { Factor de riesgo }}{\text { Clase social }^{*}}$} & \multirow{3}{*}{\begin{tabular}{l}
\multicolumn{2}{c}{ Categorías } \\
Clase $1[1]$ \\
Clase $3[2]$
\end{tabular}} & \multirow{3}{*}{$\begin{array}{c}\text { Tasa } \\
0.242 \\
0.509\end{array}$} & \multicolumn{2}{|c|}{ Observado } & \multirow{3}{*}{$\begin{array}{c}\text { IC 95\% } \\
0.353,0.642 \\
0.207,0.460\end{array}$} & \multirow{3}{*}{$\frac{\mathrm{Chi}^{2} \text { Yates }}{35.73 p<0.000}$} & \multirow{3}{*}{$\begin{array}{c}\mathrm{Chi}^{2} \text { Pearson } \\
36.79 p<0.000\end{array}$} \\
\hline & & & $\mathrm{RR}$ & 0.476 & & & \\
\hline & & & OR & 0.309 & & & \\
\hline \multirow[t]{2}{*}{ Clase social $^{*}$} & Clase 2[1] & 0.523 & $\mathrm{RR}$ & 0.750 & $0.659,0.853$ & \multirow{2}{*}{$28.03 p<0.001$} & \multirow{2}{*}{$29.11 p<0.001$} \\
\hline & Clase 3[2] & 0.697 & OR & 0.476 & $0.362,0.627$ & & \\
\hline \multirow[t]{2}{*}{ Nivel enseña } & Infantil [1] & 0.384 & $\mathrm{RR}$ & 1.141 & $0.889,1.463$ & \multirow{2}{*}{$0.8 p=0.371$} & \multirow{2}{*}{$1.00 p=0.317$} \\
\hline & Secundaria[2] & 0.337 & OR & 1.228 & $0.820,1.839$ & & \\
\hline \multirow[t]{2}{*}{ Nivel enseña } & Primaria[1] & 0.693 & $\mathrm{RR}$ & 1.069 & $0.975,1.173$ & \multirow{2}{*}{$1.58 p=0.208$} & \multirow{2}{*}{$1.77 p=0.183$} \\
\hline & Secundaria[2] & 0.648 & OR & 1.226 & $0.908,1.655$ & & \\
\hline
\end{tabular}

Tasa = proporción en el grupo del factor de riesgo con presencia de problemas de salud mental.

Riesgo relativo $=$ Tasa $[1] /$ Tasa [2]

Odds[1] = presentes [1]/ausentes [1].

Odds[2] = presentes [2]/ausentes[2].

Odds Ratio $=$ Odds[1]/Odds[2].

dradas de Pearson=29.11 y de Yates=28.03 obtuvieron $\mathrm{p}$-value significativos ( $\mathrm{p}=0.00<0.05)$. Por tanto, el factor de riesgo clase 2 en comparación con la clase 3 fue un factor protector de problemas de salud mental en los menores (cuadro 2).

2. Nivel educativo del menor. La hipótesis de independencia entre las variables nivel educativo y salud mental se aceptó con una chi-cuadrada de Pearson de 1.907 $(p=0.385>0.05)$. Además, se calcularon los RR y OR entre los niveles de las variables anteriormente citadas corroborando la hipótesis anterior de ausencia de asociación entre ellos. Al comparar las categorías E. infantil y E. secundaria, se obtuvieron $\mathrm{RR}=1.141$ y $\mathrm{OR}=1.228$. Los límites de los ratios comprendieron al valor 1 y las Chi-Cuadradas obtuvieron una p-value no significativas $(p=0.317>0.05)$. Por tanto, el factor pertenecer al periodo de educación infantil no fue un factor de riesgo con respecto a la pertenencia al periodo de educación secundaria.

Al comparar la E. secundaria con la E. primaria se obtuvieron $\mathrm{RR}=1.069$ y $\mathrm{OR}=1.226$. Los límites de los ratios (Riesgo y Odds) comprendieron al valor 1 y las Chi-cuadradas obtuvieron una $p$-value no significativas $(p=0.183>0.05)$. Por tanto, el factor pertenencia al nivel de educación primaria no fue un factor de riesgo con respecto a la pertenencia al nivel de educación secundaria.

\section{DISCUSIÓN Y CONCLUSIÓN}

El presente trabajo destaca la importancia del análisis de la clase social ocupacional de los padres en la salud de los menores españoles, siendo aquellos trabajos del principal sustentador con categorías más bajas los más relacionados con la presencia de problemas de salud mental en los menores. Por lo contrario, son las familias con trabajos correspondientes a las clases medias y las clases más privilegiadas las que suponen un factor protector frente a aquellas clases más desfavorecidas ante la presencia de problemas de salud mental en los menores. Estos datos, obtenidos con población general de menores españoles, concuerdan con otras investigaciones internacionales que demuestran que aquellos menores con familias con un estatus socioeconómico más pobre tienen una salud física y mental peor que aquellos menores con un alto nivel socioeconómico. ${ }^{10,32-36} \mathrm{Ni}$ el efecto del sexo, la raza o la edad cambian esta asociación, siendo la educación de los padres y el nivel socioeconómico las variables más fuertemente asociadas a la aparición de problemas de salud mental en los menores. ${ }^{35}$

De este modo, el bajo nivel socioeconómico asociado a las bajas categorías del nivel social puede conllevar no solamente un salud mental negativa en los adultos sino que afecta directamente a la salud mental de los menores, ${ }^{3}$ pudiendo incluso provocar unas malas relaciones de los padres con sus hijos durante periodos de tiempo más prolongados. ${ }^{37}$ Por tanto, son las categorías laborales más elevadas las que pueden influir como un factor protector en la salud mental de los menores, demostrándose una asociación entre el estatus socio-laboral de los padres y el nivel de salud del menor, no sólo a nivel físico sino también a nivel mental. ${ }^{38}$ Del mismo modo, si se asocia una baja categoría laboral con niveles bajos de ingresos, se puede entender la relación obtenida en algunos estudios entre el nivel de pobreza de una familia y la presencia de mayores problemas de ansiedad y depresión en adolescentes y jóvenes. ${ }^{39}$ Una posible intervención para disminuir el efecto de pertenecer a una clase social baja, podría ir dirigida a la mejora educativa de los padres que pertenecen a dicha clase. Se ha comprobado que el nivel educativo de los padres también es una variable que repercute en la salud mental de los menores incluso más que el desempleo o el propio estatus laboral. ${ }^{40,41}$ Conseguir una mejora de la clase social requiere de multitud de factores externos difíciles de controlar en una intervención. Sin embargo, podría ser más efectivo buscar intervenciones públicas que garanticen vías de formación para mejorar los niveles educativos de los padres y disminuir el riesgo de padecer problemas de salud mental en los menores a su cargo. Es decir, la formación educativa sería una variable a trabajar 
para conseguir modular el impacto de la pertenencia a una clase social baja en la salud mental del menor. ${ }^{35}$ Del mismo modo, parece que una baja educación y bajos ingresos en el principal sustentador van a determinar la menor probabilidad de solicitar una consulta médica para evaluar la salud mental del menor. ${ }^{42}$ De acuerdo con otros estudios internacionales ${ }^{43}$ son necesarias políticas públicas de salud mental que propicien un acercamiento a las familias en riesgo para que puedan detectar mejor las necesidades de atención psicológica en los menores a su cargo y poder darles una atención adecuada.

Por otro lado, en este estudio se demuestra que el nivel educativo ocupado por el menor en función de su edad, no se asocia con la presencia o no de problemas de salud mental. Por tanto, no supone un factor de riesgo para la presencia de problemas mentales. Estos datos difieren de algunas encuestas nacionales que establecen una mayor puntuación total en el SDQ en menores de cuatro a 9 años, en comparación con los comprendidos entre los 10 y 15 años. ${ }^{42}$ Una posible explicación se basa en la relación entre la edad y la tipología de trastornos en los menores. Se ha comprobado cómo según avanzamos en la edad del menor aparecen menos trastornos de conducta y eliminación y más trastornos del estado de ánimo y de alimentación. ${ }^{44}$ Sin embargo, según otros autores, ${ }^{45}$ la mayoría de los trastornos mentales se diagnostican a partir de los 14 años por lo que es más probable la presencia de problemas de salud mental a partir de esa edad.

Una posible explicación a estas divergencias encontradas en los diferentes estudios, podría estar relacionada con la detección y no tanto con la presencia de problemas de salud mental. Según nuestros datos, estos problemas podrían estar por igual entre los cuatro y los 15 años. Sin embargo, es posible que, dadas las características evolutivas más llamativas de la adolescencia, sea en esta etapa donde se realicen los primeros diagnósticos y evaluaciones. Por tanto, como se demuestra en este estudio, los problemas de salud mental de menores españoles pueden encontrarse en todas las etapas educativas por igual, aunque en poblaciones no nacionales o clínicas con muestras más reducidas pueda ser más fácil encontrar mayor número de diagnósticos en la etapa de la adolescencia.

Por otro lado, aunque los niveles educativos pueden no afectar en la infancia y adolescencia, se ha demostrado que en la etapa adulta, aquellas personas con mayores niveles educativos alcanzados no sólo tienen una menor probabilidad de presentar problemas de salud mental, ${ }^{46}$ sino que ante una situación de enfermedad mental tienen mayor probabilidad de acudir a un especialista para encontrar un tratamiento adecuado. ${ }^{47}$ Del mismo modo, aquellos padres con mayor formación académica informan de menores síntomas negativos de salud mental en sus hijos. ${ }^{48}$ De este modo, aquellas personas con menor nivel educativo tienen menor probabilidad de acudir a un psiquiatra, un médico, un psicólogo o un trabajador social independientemente de los ingresos económicos que obtengan, ${ }^{49}$ pudiendo afectar también en la salud del menor. Por tanto, aunque el nivel educativo puede ser un factor importante para una buena salud mental, parece que estas diferencias no aparecen en los menores españoles. Sin embargo, los estudios demuestran que es más adelante, en la etapa adulta, donde el nivel educativo va a influir en la comprensión de los problemas de salud mental y en la búsqueda adecuada de su tratamiento. ${ }^{50}$

Desgraciadamente se han encontrado más variables de riesgo relacionadas con los problemas de salud mental del menor. ${ }^{51}$ Estas variables serían: problemas de salud mental de los padres, sucesos vitales estresantes del menor (problemas médicos, problemas en la escuela, situaciones de maltrato infantil, problemas conyugales paternos), nivel de estudios bajo de los padres y, por supuesto, el estatus socioeconómico analizado en este estudio. Por todo ello, es determinante continuar con el estudio de la salud en los menores y procurar que las desigualdades en las etapas tempranas dejen de ser predictores de una mala salud en la etapa adulta. ${ }^{52,53}$ Así será fundamental conocer en profundidad cada una de las variables más influyentes en la aparición de problemas de salud mental en los menores. De este modo, se podrá conseguir una adecuada detección y un tratamiento preventivo que logre disminuir otros factores asociados a los problemas de salud mental como el abandono escolar, el abuso de sustancias, la violencia familiar y los suicidios. ${ }^{54,55}$

\section{Financiamiento}

Ninguno.

\section{Conflicto de intereses}

Los autores declararon no tener conflicto de intereses.

\section{REFERENCIAS}

1. Boardman JD, Alexander KB, Miech RA, MacMillan $R$ et al. The association between parent's health and the educational attainment of their children. Soc Sci Med 2012;75:932-939.

2. Bradley RH, Corwyn RF. Socioeconomic status and child development. Annu Rev Psychol 2002;53:371-399.

3. Bones K, Pérez K, Rodríguez-Sánza M, Borrell C et al. Prevalencia de problemas de salud mental y su asociación con variables socioeconómicas, de trabajo y salud: resultados de la Encuesta Nacional de Salud de España. Psicothema 2010;3:389-395.

4. Mackenbach JP, Martikainen P, Looman C, Dalstra J et al. The shape of the relationship between income and self-assessed health: an international study. Int J Epidemiol 2005;34:286-293.

5. Marmot M. Status syndrome: How your social standing directly affects your health and life expectancy. Londres: Bloomsburry Publishing; 2004.

6. Wilkinson R, Pickettt K. The spirit level: Why equality is better for everyone. Londres: Penguin Books; 2010.

7. Alegria M, Bijl RV, Lin E, Walters EE et al. Income differences in persons seeking outpatient treatment for mental disorders: A comparison of the United States with Ontario and the Netherlands. Arch Gen Psychiatry 2000;57:383-391. 
8. Wang PS, Berglund PA, Kessler RC. Recent care of common mental disorders in the United States: Prevalence and conformance with evidence-based recommendations. J Gen Intern Med 2000;15:284-292.

9. Chen E, Martin AD, Matthews KA. Socioeconomic status and health: do gradients differ within childhood and adolescence? Soc Sci Med 2006;62:2161-2170.

10. Chen E, Matthews KA, Boyce WT. Socioeconomic differences in children's health: How and why do these relationships change with age? Psychol Bull 2002;128:295-329.

11. Bourdon KH, Goodman R, Rae DS, Simpson G et al. The strengths and difficulties questionnaire: Normative data and psychometric properties. J Am Acad Child Adolesc Psychiatry 2005;44:557-564.

12. Egger HL, Angold A. Common emotional and behavioral disorders in preschool children: Presentation, nosology, and epidemiology. J Child Psychol Psychiatry 2006;47:313-337.

13. Zwirs BW, Burger $H$, Schulpen TW, Wiznitzer $M$ et al. Prevalence of psychiatric disorders among children of different ethnic origin. J Abnorm Child Psychol 2007;35:556-566.

14. Fonseca-Pedrero E, Paino M, Lemos-Giráldez S, Muñiz J. Prevalencia de la sintomatología emocional y comportamental en adolescentes españoles a través del strengths and difficulties questionnaire (SDQ). RPPC 2011;16:15-25.

15. Goodman R. The strengths and difficulties questionnaire: a research note. J Child Psychol Psychiatry 1997;38:581-586.

16. Muris $P$, Meesters $C$, Van den Berg $F$. The strengths and difficulties questionnaire (SDQ). Further evidence for its reliability and validity in a community sample of Dutch children and adolescents. Eur Child Adolesc Psychiatry 2003;1:1-8.

17. Vostanis P. Strengths and difficulties questionnaire: research and clinical applications. Curr Opin Psychiatry 2006;19:367-372.

18. Gomez-Beneyto $M$, Nolasco A, Moncho J, Pereyra-Zamora $P$ et al. Psychometric behavior of the strengths and difficulties questionnaire (SDQ) in the Spanish National Health Survey 2006. BMC Psychiatry 2013;13:95. doi: 10.1186/1471-244X-13-95

19. Goodman A, Goodman R. Population mean scores predict child mental disorder rates: validating SDQ prevalence estimators in Britain. J Child Psychol Psychiatry 2011;52:100-108.

20. Goodman R, Scott S. Comparing the strengths and difficulties questionnaire and the child behavior checklist: is small beautiful? J Abnorm Child Psychol 1999;27:17-24.

21. Maso WA, Chmelka MB, Thompson RW. Responsiveness of the strengths and difficulties questionnaire (SDQ) in a sample of high -risk youth in residential treatment. Child Youth Care Forum; 2012; http:// dx.doi.org/10.1007/s10566-012-9179-5

22. Rodríguez PJ, Betancort M, Ramírez GM, García R et al. Psychometric properties of the parent and teacher versions of the strengths and difficulties questionnaires (SDQ) in a Spanish sample. Int J Clin Health Psycchol 2012;12:265-279.

23. Fajardo F, León B, Felipe E, Santos E. Salud mental en el grupo de edad de 4 a 15 años a partir de los resultados de la encuesta nacional de salud 2006. Rev Esp Salud Publica 2012;86:445-451.

24. Ministerio de Sanidad y Política Social. Encuesta na 7 cional de salud 2006. Consultado el 20 de junio de 2015. Disponible en: http://www. msssi.gob.es/estadEstudios/estadisticas/encuestaNacional/encuesta2006.htm

25. Giannakopoulos G, Tzavara C, Dimitrakaki C, Kolaitis G et al. The factor structure of the strengths and difficulties Questionnaire (SDQ) in Greek adolescent. Ann Gen Psychiatry 2009;8:20. http://dx.doi.org/10.1186/1744-859X-8-20

26. Stone L, Otten R, Engels R, Vermulst AD et al. Psychometric properties of the parent and teacher versions of the strengths and difficulties questionnaire for 4- to 12-year-olds: a review. Clin Child Fam Psychol Rev 2010;13:254-274.

27. Ullebo AK, Posserud MB, Heiervang E, Gillberg C et al. Screening for the attention deficit hyperactivity disorder phenotype using the strength and difficulties questionnaire. Eur Child Adolesc Psychiatry 2011;20:451-458.

28. Van Widnelfelt BM, Goedhart AW, Treffers PDA, Goodman R. Dutch version of the strengths and difficulties questionnaire (SDQ). Eur Child Adolesc Psychiatry 2003;12:281-289.

29. Goodman R, Renfrew D, Mullick M. Predicting type of psychiatric disorder from strengths and difficulties questionnaire (SDQ) scores in child mental health clinics in London and Dhaka. Eur Child Adolesc Psychiatry 2000;9(2):129-134.

30. Goodman A, Goodman R. Population mean scores predict child mental disorder rates: validating SDQ prevalence estimators in Britain J Child Psychology Psychiatry 2011;52(1):100-108.

31. Domingo-Salvany A, Regidor E, Alonso J, Alvarez-Dardet C. Proposal for a social class measure. Working Group of the Spanish Society of Epidemiology and the Spanish Society of Family and Community Medicine. Aten Primaria 2000;25:350-363.

32. Duncan GJ, Brooks-Gunn J. Consequences of growing up poor. New York: Russell Sage Foundation; 1997.

33. Leventhal T, Brooks-Gunn J. The neighborhoods they live in: The effects of neighborhood residence on child and adolescent outcomes. Psychol Bull 2000;126:309-337.

34. Starfield B, Robertson J, Riley AW. Social class gradients and health in childhood. Ambul Pediatr 2002;2:238-246.

35. Perna L, Bolte G, Mayrhofer H, Spies G et al. The impact of the social environment on children's mental health in a prosperous city: an analysis with data from the city of Munich.BMC Public Health 2010;10:199. doi: 10.1186/1471-2458-10-199

36. Starfield B, Riley AW, Witt EP, Robertson J. Social class gradients in health during adolescence. J Epidemiol Community Health 2002;56:354-361.

37. Kroenke C. Socioeconomic status and health: Youth development and neomaterialist and psychosocial mechanisms. Soc Sci Med 2008; 66:3142.

38. Siponen SM, Ahonen RS, Savolainen PH, Hämeen-Anttila K. Children's health and parental socioeconomic factors: a population-based survey in Finland. BMC Public Health, 2011;11:457. http://dx.doi.org/ doi:10.1186/1471-2458-11-457

39. Najman JM, Hayatbakhsh MR, Clavarino A, Bor W. Family poverty over the early life course and recurrent adolescent and young adult anxiety and depression: a longitudinal study. American Public Health 2010;100:1719-1723.

40. Boe T, Overland S, Lundervold AJ, Hysing M. Socioeconomic status and children's mental health: results from the Bergen child study. Soc Psychiatry Psychiatr Epidemiol 2012; 47:1557-1566.

41. Reiss F. Socioeconomic inequalities and mental health problems in children and adolescents: A systematic review. Soc Sci Med 2013; 90:24-31.

42. Haines M, McMunn J, Nazroo J, Ivonne J. Social and demographic predictors of parental consultation for child psychological difficulties. J Public Health Med 2002; 24(4):276-284.

43. Hölling H, Bärbel-Maria K, Rothenberger A, Becker A et al. Assessing psychopathological problems of children and adolescents from 3 to 17 years in a nationwide representative sample: results of the German health interview and examination survey for children and adolescents (KiGGS). Eur Child Adolesc Psychiatry 2008; [Suppl1]17:34-41.DOI 10.1007/s00787-008-1004-1

44. Navarro-Pardo E, Meléndez JC, Sales A, Dolores M. Desarrollo infantil y adolescente: trastornos mentales más frecuentes en función de la edad y el género. Psicothema 2012;24(3):377-383.

45. Kessler RC, Berglund P, Demler O, Jin R et al. Lifetime prevalence and age-of-onset distributions of DSM-IV disorders in the National Comorbidity Survey Replication. Arch Gen Psychiatry 2005; 62(6):593602.

46. Braveman P, Egerter S, Williams D. The social determinants of health: Coming of age. Annu Rev Publ Health 2011; 32:381-398. 
47. Sanders LM, Federico S, Klass P, Abrams MA et al. Literacy and health outcomes: a systematic review. Arch Pediatr Adolesc Med 2009; 163:131-40.

48. Sonego M, Llácer A, Galán I, Simón F. The influence of parental education on child mental health in Spain. Qual Life Res 2013; 22:203-211.

49. Steele LS, Dewa CS, Lin E, Lee KL. Education level, income level and mental health services use in Canada: Associations and policy implications. Health Policy 2007; 31:96-106.

50. Yen CF, Chen CC, Lee Y, Tang TC et al. Insight and correlates among outpatients with depressive disorders. Compr Psychiatry 2005;46:384389.

51. Mendes AV, Souza JA, Souza RM, Loureiro SR. Risk factors for mental health problems in school-age children from a community sample. Matern Child Health J 2013; 17(10):1825-1834.
52. Kestilä L, Koskinen S, Martelin T, Rahkonen $O$ et al. Determinants of health in early adulthood: what is the role of parental education, childhood adversities and own education? Eur J Public Health 2005; 16:305-314.

53. Melchior M, Moffitt TE, Milne BJ, Poulton R et al. Why do children from socioeconomically disadvantaged aamilies suffer from poor health when they reach adulthood. A life-course study. Am J Epidemiol 2007; 166:966-974.

54. Access Economics. The economic impact of youth mental illness and the cost effectiveness of early intervention. Canberra: 2009.

55. Beardslee WR, Wheelock I. Children of parents with affective disorders: Empirical findings and clinical implications. En: Handbook of depression in children and adolescents. New York; Plenum; 1994; pp.463-479. 